\title{
Les valeurs (in)différentes entre identité et image: le cas Trenitalia ${ }^{1}$
}

Michela Deni

Institut ACTE (UMR 8218, CNRS/Université Paris 1)

Équipe «Sémiotique des Arts et du Design», groupe PROJEKT Université de Nîmes

michela.deni@gmail.com

\begin{abstract}
Cette intervention propose une réflexion sur les concepts d'identité et valeurs, pour arriver à leur application et à l'exemplification à travers le cas spécifique d'une entreprise, Trenitalia.

Dans la première partie de l'article nous aborderons les concepts de valeurs et valorisation. Ensuite, la seconde partie de l'article sera consacrée à l'importance de la thématisation et de la manifestation de valeurs en montrant comment ces deux passages sont délicats et décisifs pour construire l'identité d'une entreprise: en effet, le manque de conscience dans la gestion du processus entier de valorisation peut mener une entreprise à gâcher ses objectifs en compromettant ses intentions tant communicatives que éthiques. Dans la troisième partie de l'article nous définirons le concept d'identité d'entreprise. À titre d'exemple, la dernière partie de l'article $(\S 4,5)$ établira une comparaison dans la gestion de l'image d'entreprise entre Trenitalia, Italo Nuovo Trasporto et Sncf.
\end{abstract}

Using the concepts of identity and values as a starting point, our paper introduces the example of the company Trenitalia to explain the differences between Corporate Identity and Corporate Image.

In the first part of the paper, we define values and valorization. Then, the second part, deals with the passage between "themes" and "manifestations" of values by demonstrating how this transposition is decisive to build the identity of a company. In fact, a considerable gap between the mission of a company and the manifestations concerning this company (products, services, communication, acts, etc.) could compromise its communicative intentions and its credibility. In the third part of the paper, we propose some examples to define the Corporate Identity and the Corporate Image. In particular, in the last paragraphs we compare how Trenitalia, Italo Nuovo Trasporto and SNCF build and manage their Corporate Image.

\section{Keywords}

Identità di marca, Valori, Semiotica, Corporate Identity, Corporate Image, values, semiotics

\footnotetext{
${ }^{1}$ Une première version de cette intervention a été présentée le 18/o9/12 en tant que conférence introductive dans le séminaire C'est l'affaire de tous! Identité \& valeurs en entreprise, organisée par S. d'Armagnac, J.-C. Benech, R. Dardel, M. Deni et D. Galarreta au CCT MAN Cnes Toulouse, IAS.
} 


\section{Oculartux_saggi}

Les valeurs (in)différentes entre identité et image: le cas Trenitalia | Michela Deni

\section{Les valeurs en amont}

Chaque entreprise se présente à travers sa communication, mais surtout à travers ses produits, ses services et les personnes qui y travaillent.

Les valeurs implicites autant que les valeurs déclarées à partir de "la mission" constituent l'identité profonde d'une entreprise et nous permettent de l'identifier dans le panorama des entreprises nationales et internationales.

Parfois il y a un écart assez important entre ce que l'entreprise déclare et la manière dont le sentiment public général la reconnait: cette différence nous permet de distinguer l'identité d'entreprise par rapport à son image. ${ }^{2}$ En cas de distance flagrante entre ces deux grandeurs, effet qui peut se produire dans le temps, le management d'entreprise devrait identifier les dissonances qui peuvent survenir entre ces deux valeurs en jeu, et cela afin de les faire converger et gagner à nouveau la confiance de son public.

Tous parlent de valeurs, d'après le sens commun de quoi s'agit-il?

Définition de valeur:

Ce qui est posé comme vrai, beau, bien, d'un point de vue personnel ou selon les critères d'une société et qui est donné comme un idéal à atteindre, comme quelque chose à défendre. ${ }^{3}$

En général et selon ce sens plus répandu, la valeur est donc considérée en termes axiologiques, c'est-à-dire comme quelque chose qui est "bien", qui est culturellement et socialement partagée: en d'autres termes la valeur est donnée et il suffit seulement d'agir pour la respecter, pour s'y conformer et ainsi l'atteindre.

Mais essayons de penser autrement, en oubliant un peu la généralité du sens commun en proposant plutôt une réflexion préliminaire sur la valeur plus proche à la sémiotique.

Qu'est-ce qu'une valeur avant qu'elle ne soit devenue une valeur ?4 Nous pouvons répondre que c'est un mot, plus précisément son signifié, autrement-dit une idée. Cette base sémantique pourrait être considérée comme neutre. Ainsi, sont les mots qui reçoivent une définition dans le dictionnaire et qui, en principe, peuvent être considérés sans attribution particulière (femme, homme; innovation, tradition; numérique, analogique; rapidité, lenteur; futur, passé; vie, mort; etc.), prenant au cours du temps, des connotations affectives et valorielles. Ces valorisations sont inscrites à partir de la langue et de la culture d'appartenance.

Chaque mot, ainsi que chaque catégorie sémantique, avant d'être investis des valeurs, pourraient être considérés comme neutre. Une même unité, par exemple le mot environnement, ou écologie, identifierait une même unité de

2 À ce sujet cf. Bianchi et Ragonese 2013; Floch 1990, 1995; Fabris et Minestroni 2004; Marrone 2007; Musso 2013, Semprini 2005, Heilbrunn 2010.

3 À l'entrée «valeur»: www.larousse.fr/dictionnaires/francais/valeur/80972?q=valeur

4 Sur valeur et valorisation voir Greimas 1970, 1983; Greimas et Courtés 1979; Greimas et Courtés (éds.) 1986. 


\section{Oculartux_saggi}

Les valeurs (in)différentes entre identité et image: le cas Trenitalia | Michela Deni

sens à des époques ou dans des cultures différentes. L'investissement des valeurs - en d'autres termes la valorisation - peut être alors saisi comme un processus qui vise à connoter comme euphorique (positif pour le but d'un ou plusieurs sujets) ou comme dysphorique (négatif pour le but d'un ou plusieurs sujets).

On peut affirmer qu'une des finalités des discours est de changer les connotations de ces mots que nous appelons "valeurs". Toute entreprise accomplie des opérations: la première est celle de s'approprier ces mot-valeurs, par exemple en les référant à son histoire à ses produits ou à son image; la seconde est de maîtriser et faire varier leur charge valorielle.

Dans les valeurs d'une entreprise, par exemple, le futur ou le passé peuvent ne pas être marqués par une valeur spécifique mais le devenir en revanche dans un contexte donné (culturel, industriel, de marchandise).

Comment se produit donc ce procès d'appropriation, d'identification et de promotion?

\subsection{Les valeurs interdéfinies}

Les valeurs n'existent pas en elles mêmes et ne sont ni positives ni négatives mais elles se déterminent les unes par rapport aux autres. En d'autres termes, toute valeur « est une valeur positionnelle »: les valeurs n'ont pas d'existence isolée et se définissent réciproquement. En outre, cette charge valorielle peut être positive ou négative. 5

Pourtant, en termes axiologiques, chaque valeur peut en même temps être positive dans un domaine, neutre dans un autre domaine ou enfin négative dans un dernier contexte (l'innovation? la tradition? le développement soutenable? le luxe? la santé?). On pourrait conclure alors que chaque valeur ne devient valeur qu'au moment où elle est investie de façon positive ou négative par un discours donné. Et c'est alors que la rhétorique prend en charge cette mise en valeur. Le procès nécessaire aux valeurs est donc l'investissement de la charge plus ou moins positive ou négative. Nous l'appellerons valorisation, ou mise en valeur.

La valorisation est l'opération stratégique fondamentale pour la constitution de valeurs. Pour cela devient alors fondamental, dans une entreprise, la capacité de maîtriser les opérations de valorisation à chaque niveau.

En ce sens, le but du management d'entreprise n'est pas simplement celui d'identifier et de construire les valeurs, mais aussi: de traduire ces valeurs en les adaptant à l'actualité; les rendre spécifiques même quand elles sont partagées par la majorité des entreprises qui peuvent les décliner différemment (je pense, en cela, au développement durable); ou bien les rendre cohérentes entre-elles dans les cas qui pourraient devenir contradictoires comme par exemple la valeur de la recherche évoquée par

\footnotetext{
5 Nous renvoyons au concept de valeur linguistique introduit par De Saussure. En ce termes, « le sens ne réside que dans les différences saisies entre les mots [...] en termes de valeurs, relatives », entrée « Valeur » dans Greimas et Courtés 1979.
} 


\section{Oculart_r_saggi}

Les valeurs (in)différentes entre identité et image: le cas Trenitalia | Michela Deni

plusieurs entreprises (l'éthique de la recherche? la recherche pour le profit? le profit finalisé à la recherche?, etc.).

\subsection{Valeurs et identité en entreprise}

Les valeurs sont les paliers sur lesquelles se fonde - et se déclare - le discours d'une entreprise. En d'autres termes: les valeurs construisent et constituent le noyau et la raison d'être d'une entreprise en devenant la partie constitutive de son " identité éthique », c'est-à-dire le contrat de confiance profond avec lequel une entreprise se présente et qu'elle, pour être reconnue de façon durable, s'engage à maintenir dans le temps. ${ }^{6}$

En cas de problèmes et surtout lorsqu'il s'agit des entreprises avec une mission de service à participation publique, leur image risque d'être gravement compromise à cause de la rupture du contrat de confiance basé sur les valeurs de l'entreprise et implicitement stipulé entre l'entreprise, ses employés et le public. Donc, la conception des produits, des services ainsi que leurs territoires d'intervention à prévoir ${ }^{7}$ (c'est-à-dire les domaines d'évolution de l'entreprise) doit être conçue d'une façon cohérente à partir de la mission déclarée et prise en charge par l'entreprise en tant que vrai contrat éthique.

\subsection{Les valeurs de base et les valeurs d'usage}

La sémiotique narrative nous fournit une distinction importante entre les valeurs de base (but) et les valeurs d'usage (moyen). Cette démarcation est à poser chaque fois et nous devient utile pour réfléchir au parcours de valorisation, implicite ou explicite, propre à chaque entreprise.

Ainsi pour une entreprise donnée, le développement durable pourrait représenter le but (valeur de base), ou bien pourrait être le moyen pour atteindre d'autres objectifs (valeur d'usage). Mais en même temps, ce qui pour l'entreprise pourrait être le but (recyclage des matériaux), pour les clients pourrait être en revanche juste le moyen d'atteindre un autre but (l'épargne).

Sur les valeurs de base (la raison d'être de l'entreprise) se jouent la planification des projets, l'organisation et la hiérarchie des étapes à suivre (les valeurs virtuelles, actualisées et réalisées), les stratégies de communication. Les valeurs d'usage doivent alors être cohérentes avec les valeurs de base et ces deux catégories de valeurs doivent se mettre en parallèle en permanence.

${ }^{6} \mathrm{Cf}$. Semprini 2005 .

7 Pour une définition de territoires voir Semprini 2005. 


\section{Oculartux_saggi}

Les valeurs (in)différentes entre identité et image: le cas Trenitalia | Michela Deni

\section{Des valeurs aux thèmes, des thèmes aux manifestations}

\subsection{Les Thèmes}

Dans l'entreprise, une fois les valeurs de base établies, l'étape suivante pour les concrétiser sera de les détailler: par exemple le développement durable pourra être thématisé par le tri sélectif des déchets; par le recyclage des matériaux; par l'utilisation de matériaux entièrement naturels et biodégradables; ou encore par l'usage de panneaux solaires.

En d'autres termes, le thème est la façon dans laquelle les valeurs commencent à prendre forme. ${ }^{8}$

Inutile de dire que le choix du thème est contraignant par rapport à ce qu'une entreprise voudrait communiquer et, en même temps, par rapport à ce qui est compris par les clients, ou, par la collectivité. Une thématisation inadaptée peut compromettre l'interprétation des valeurs en affirmant implicitement d'autres valeurs dévoilant clairement l'intention élitiste et liée au profit de l'entreprise - comme c'est le cas pour Trenitalia (§ 4.4).

\subsection{Manifestations}

Une fois les valeurs établies (implicitement ou non), les thèmes pour les manifester choisis, il sera temps de les expliciter et concrétiser avec des produits, des services, des images, des textes, des prix et de la communication.

Pour une entreprise, les valeurs doivent coïncider avec ses motivations profondes d'exister et d'opérer dans le marché. Il s'agit des motivations qui poussent le management d'entreprise dans son choix concernant la production (produits, service) le prix, les espaces de vente, les employés, le site, le service clients, le service après-vente, la gestion, l'organisation du travail et la communication. Les valeurs d'entreprise seront concrétisées à travers chaque choix de l'entreprise, en d'autres termes, par l'ensemble de ses manifestations.

La communication de l'entreprise est alors une des manifestations de l'entreprise, certainement importante, mais demeurant toujours l'une d'entre elles. Le jugement de cohérence passe donc non seulement par la communication, mais aussi, et surtout, par cet ensemble de manifestations. ${ }^{9}$ Pour mettre en relief ses valeurs, l'entreprise agira selon chaque contexte en trouvant la façon de les représenter et de les raconter en unifiant toutes les exigences de toutes les composantes et les partenaires en jeux.

\footnotetext{
${ }^{8}$ En fait « on peut définir le thème comme la dissémination, le long des programmes et parcours narratifs, des valeurs déjà actualisées [...] par la sémantique narrative. », entrée « Thème » dans Greimas et Courtés 1979.

${ }_{9}^{9}$ Semprini (2005) décrit en détail ce processus, notamment dans le modèle « Projet Manifestations » qu'il propose.
} 


\section{Oculartux_saggi}

Les valeurs (in)différentes entre identité et image: le cas Trenitalia | Michela Deni

\section{Identité entre permanence et dynamisme}

Pourquoi abordons-nous l'identité après les valeurs? Cela peut s'expliquer par le fait que les valeurs déclinées pour chaque domaine de manifestations deviennent les paliers qui fondent la construction de l'identité de l'entreprise. La question de l'identité est fondamentale pour chaque management de projet. Ainsi dans chaque entreprise, le management doit il identifier minutieusement les valeurs qui la distinguent pour construire son identité reconnaissable. Lorsque l'identité de l'entreprise fonctionne bien, il faut la maintenir dans le temps en apportant des changements cohérents qui puissent être rapportés à cette identité profonde, ou bien qui puissent la transformer graduellement, si le management le souhaite. Pourtant, il ne faut jamais oublier que chaque identité, pour garantir son caractère de permanence, est dynamique. Prendre conscience de la complexité et de la stratification du concept d'identité d'une entreprise est fondamental. Cette conscience doit être accompagnée d'une autre conscience: celle de la communication de l'identité souhaitée. Communication dans le sens vaste du terme parce qu'elle concerne chaque manifestation d'une entreprise. Au-delà du logo, du site, de la pub, des produits et tous les supports construits pour communiquer, lidentité d'une entreprise passe à travers et autour de tout ce qui concerne cette entreprise, comme elle passe aussi par les "actes" qui ne sont pas conçus pour communiquer mais qui - malgré tout - constituent sa communication involontaire. Ce dernier aspect n'est pas moins important.

À quoi travaillons-nous lorsque nous sommes appelés à analyser ou construire l'identité d'une entreprise? Nous travaillons, avant tout, à faire prendre conscience du fait qu'il est impossible de ne pas communiquer en permanence, en particulier quand il s'agit de grandes entreprises avec des événements continus, qui sont sous les yeux d'un public toujours plus vaste et plus hétérogène que ce que l'on avait prévu ou espéré rejoindre. C'est à partir de cette communication autant imprévue qu'inévitable que l'entreprise dévoile son identité ainsi que sa cohérence avec les valeurs déclarées par «sa mission », c'est à dire ses raisons d'être, raisons qui légitiment son existence aux yeux des clients et des citoyens.

Afin de mieux réfléchir sur la construction de l'identité d'une entreprise, il nous faut aussi introduire une différence opérative utile à saisir, la différence entre «identité » et « image ». Selon certains chercheurs du champs d'étude consacré au marketing, l'identité est la Corporate Identity et concerne les valeurs établies par l'entreprise (ce que l'entreprise dit); en revanche, l'image, la Corporate Image, fait référence à ce qui est ressenti par le public à propos d'une entreprise (aussi bien que par les discours sur l'entreprise même: réseaux sociaux, blogs, presse, télé, etc.). Le but de chaque entreprise est d'obtenir un degré de cohérence soutenable entre son identité déclarée et son image perçue.

Pour résumer, les valeurs fondent l'identité de l'entreprise tandis que les manifestations nous montrent son image: image comme représentation et 


\section{Ocula ${ }^{\text {flux_saggi }}$}

Les valeurs (in)différentes entre identité et image: le cas Trenitalia | Michela Deni

aussi comme miroir important pour l'entreprise elle-même (donc très utile au management quand ce dernier est bien à l'écoute). ${ }^{10}$

\section{Trenitalia entre identité et image}

Trenitalia est un cas plutôt exemplaire de divergence entre identité et image d'entreprise. Il s'agit d'une entreprise qui depuis plusieurs années n'est pas à l'écoute de ces divergences, ou presque désintéressée à les affronter à cause de son quasi monopole.

En 2011 NTV Italo est arrivé sur le marché italien en rencontrant d'une part des difficultés politiques (qui ont pris la forme d'obstacles bureaucratiques, structurales et logistiques), et d'autre part la confiance des voyageurs bien avant son arrivée dans le panorama de transports publiques.

\subsection{Les valeurs de Trenitalia}

Si l'on se concentre sur la mission déclarée de Trenitalia, nous trouvons les valeurs auxquelles on s'attend: innovation technologique; sécurité; qualité de la vie de voyageurs et des employés; futur; respect de l'environnement; solidarité.

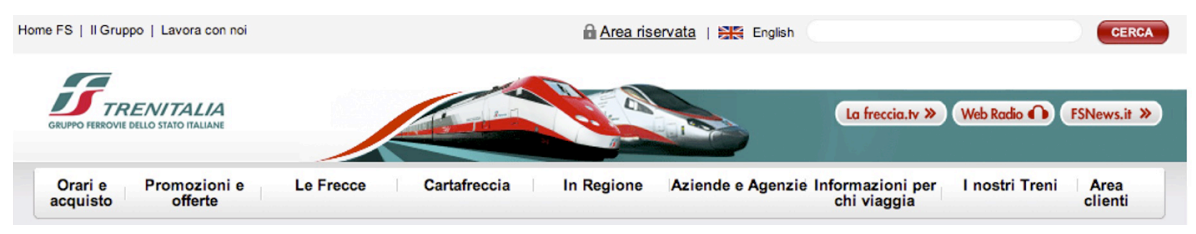

Image 1. <www.acquistionline.trenitalia.it/esop/toolkit/initRegistration.do>.

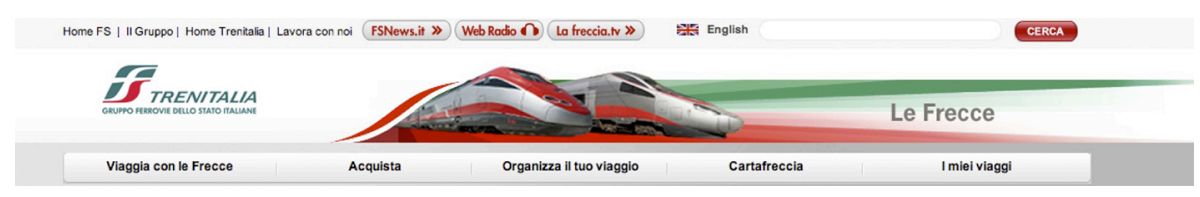

Image 2. <www.trenitalia.com> (page consultée le 16/12/14).

\subsection{Valeurs de NTV Italo}

NTV Italo investit sur les différences avec Trenitalia en se positionnant d'une façon complémentaire et, en même temps, opposée, proposant:

Services spécifiques pour les exigences des clients

Confort et technologie dans les voitures

Qualité et excellent rapport qualité/prix

${ }^{10} \mathrm{Cf}$. Semprini 2005. 


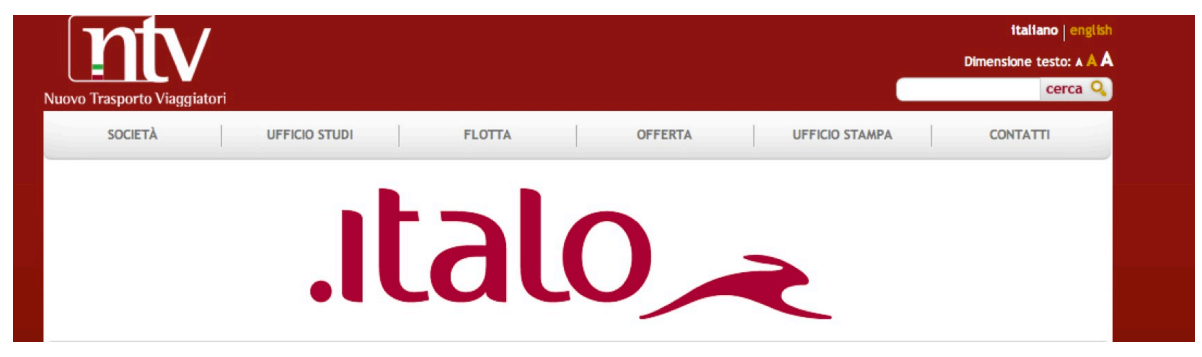

Image 3. <www.italotreno.it/IT/Pagine/default.aspx>.

4.3 Les valeurs de Trenitalia: quelques exemples à partir des manifestations

Les services (les lignes, la fréquence, le confort montrent déjà les priorités de l'entreprise), les produits (les TGV Frecciarossa et les trains régionales), les prix, la pub ainsi que les retards et le manque de communication, en cas de problèmes ou de retards, sont des manifestations fort contrastées par rapport à l'identité déclarée par Trenitalia. Même l'impossibilité d'ajouter des commentaires sur les vidéos officielles postées sur le site YouTube est une manifestation importante par rapport à la construction de l'image des clients qui s'oppose à l'identité déclarée (« qualité de la vie de voyageurs »).

Ces manifestations sont les moyens à travers lesquelles Trenitalia exprime dans la pratique ses valeurs (qualité, technologie, confort, etc.). Voilà pourquoi une entreprise peut très bien communiquer ses propres valeurs, mais c'est lors de l'incarnation de la prise en charge de ces valeurs par la pratique, qu'elle montre l'état réel des choses au delà de toutes affirmations ou bien de toutes déclarations de mission.

Non seulement les clients ridiculisent certaines annonces aux haut-parleurs répétées depuis des années ("merci d'avoir choisi Trenitalia"), mais l'impression de devenir des otages - par l'absence d'alternatives comme cela existait depuis quelques années - et le manque de confiance augmentent face aux contradictions évidentes entre la réalité des voyageurs et la mission déclarée à partir de la communication officielle.

Il s'agit d'un cas dans lequel l'impression donnée renvoie à une entreprise dont la nécessité est de camoufler le vide éthique par le biais de la communication

\subsection{La valeur, du thème à la manifestation}

Dans les TGV Frecciarossa, Trenitalia a aboli la première et la seconde classe pour proposer, en utilisant un euphémisme, quatre nouveaux types de service (ou bien restaurer quatre classes, comme disent plutôt les mauvaises 


\section{Oculatiux_saggi}

Les valeurs (in)différentes entre identité et image: le cas Trenitalia | Michela Deni

langues): Standard, Premium, Business, Executive. Il s'agit de quatre niveaux de tarifs qui proposent des services divers ainsi qu'une qualité de voyage différente. Alors que les valeurs de "qualité" et de "confort" pouvaient être présentées par l'entreprise de plusieurs façons dans le tarif standard, elles ont été argumentées et thématisées, par le choix "voyage en famille en TGV". Ce "thème" - comme chaque thème - avait la possibilité de se traduire en montrant plusieurs types de familles (parents jeunes, famille nombreuse ou n'importe quelle famille qui aime voyager avec le confort à petit prix). Et c'est là que Trenitalia a construit son discours (une pub audiovisuelle dans ce cas) en provoquant sur les médias un débat fort contrasté qui montre que les manifestations risquent de détourner l'interprétation des valeurs.

La première publicité (2012) a été qualifiée dans la presse de "pub raciste de Trenitalia, les immigrés en quatrième classe" et Trenitalia l'a retirée comprenant que les valeurs à communiquer étaient largement ratées.

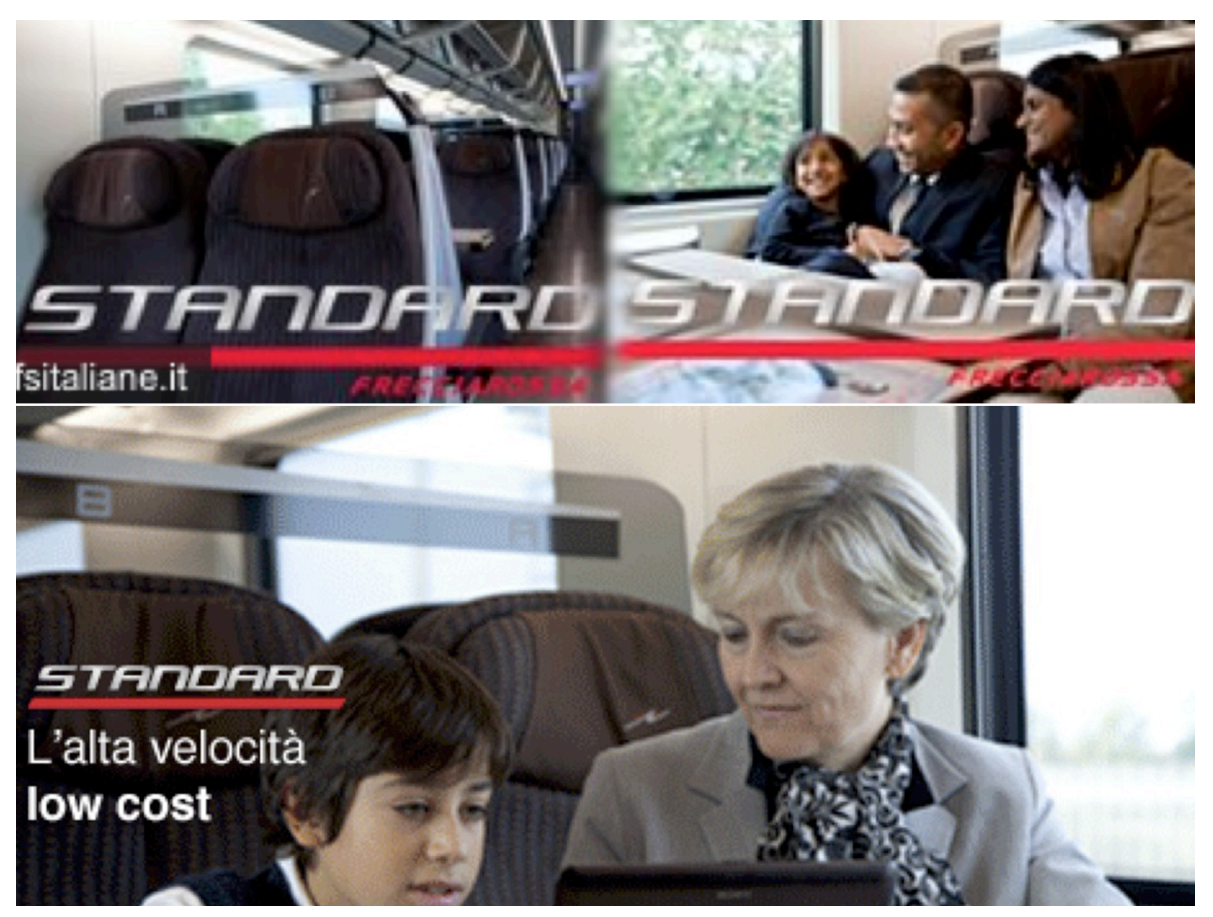

Images 4.-5.

<www.repubblica.it/cronaca/2012/01/o4/foto/trenitalia_il_fotogramma_dello_spot_razzista $-27585941 / 4 />$.

Lorsque Trenitalia a remplacé cette première publicité, la déclinaison de la thématique "voyage en famille" a été celle d'une grand-mère et son petit fils. Or, ce dernier choix communique que le standard représente un tarif correct pour deux sujets "non-actifs" qui ne gagnent pas d'argent, et donc qui nécessitent des petit prix. 


\section{Oculartax_saggi}

Les valeurs (in)différentes entre identité et image: le cas Trenitalia | Michela Deni

\section{L'image de Trenitalia}

De la part des médias et des voyageurs Trenitalia est accusée depuis toujours de diviser l'Italie en deux parties: le nord et le sud; les mégalopoles et les villes de province. En d'autres termes, l'innovation, la technologie et le confort (les valeurs pour le premier pôle de ces couples) contre les retards, les vieilles voitures en panne (pour le second). À lire la presse et à suivre les informations et les données diffusées par les associations des consommateurs, ainsi que par les réseaux sociaux, il y a une différence très éloquente sur Twitter, par exemple, en suivant le hashtag \#trenitalia entre les tweets officiels de l'entreprise et ceux envoyés par les voyageurs.

D'une part il y a les voyageurs postant des tweets et lançant sans arrêt des messages exaspérés, ironiques ou formulant des véritables parodies sur Trenitalia; d'autres parts les tweets officiels, diffusés par l'entreprise, qu'informent les clients sur les derniers services, innovations, promotions et conforts que la majorité de passagers qui font la navette (donc condamnés à la carence des investissements) ne perçoivent même pas. Tout compte fait, il y a une entreprise incapable de gérer le contrat de confiance avec les clients, et encore moins sa propre communication qui - en cette situation - se prête à toute sorte d'ironie.

\subsection{Les concurrents}

Sncf ou NTV-Italo en revanche n'ont pas la même divergence entre la communication de l'entreprise et les commentaires des clients:

\#SNCF

\#NTV ou @ItaloTreno

SNCF, en plus, gère avec transparence les tweets officiels de l'entreprise:

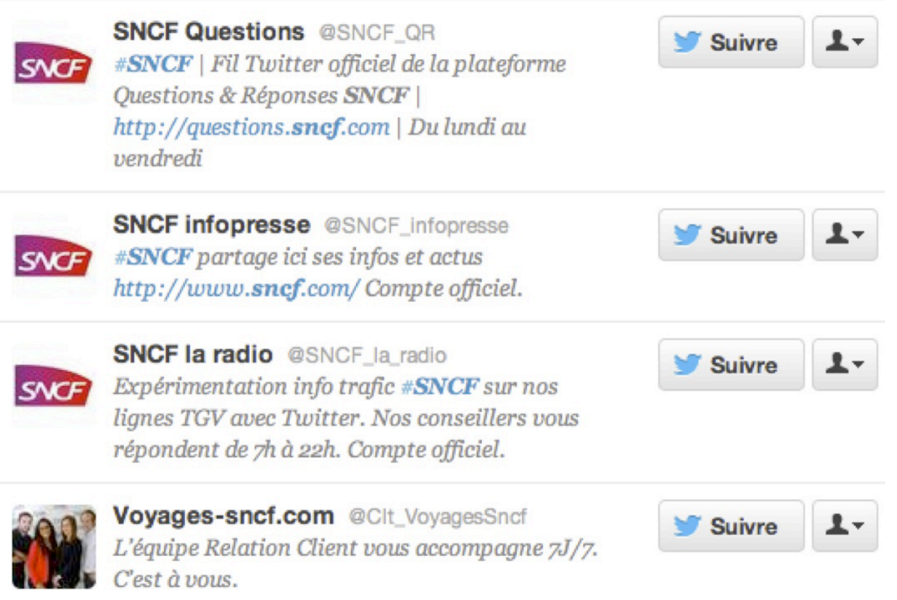

Image 6. \#sncf <www.twitter.com> (France, page consultée le 6/9/12). 


\section{Ocula ${ }^{\text {flux_saggi }}$}

Les valeurs (in)différentes entre identité et image: le cas Trenitalia | Michela Deni

Ces tweets nous permettent d'évaluer leur fréquence temporelle: les tweets postés au sujet de Trenitalia par les passagers mécontents se comptent en dizaines par heure, une quantité incomparable avec les tweets sur SNCF ou NTV-Italo, qui ne sont mentionnés que juste quelques fois par jour.

Les réseaux sociaux - comme il y a quelques années les forums - sont un bon thermomètre à surveiller par les entreprises qui veulent se questionner sur la différence qui caractérise leur identité et leur image et, de cela, elles en sont bien conscientes : ${ }^{11}$

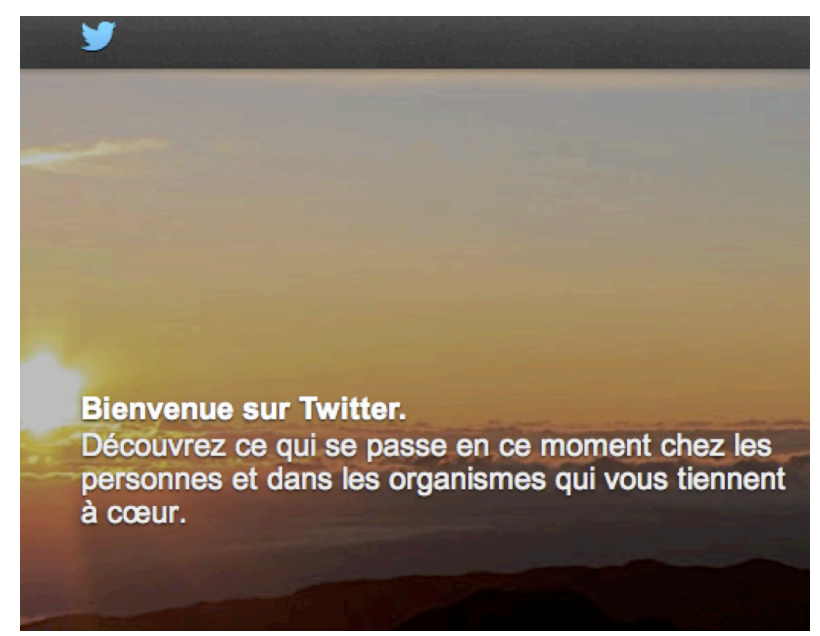

\section{Bienvenue sur Twitter.}

Connectez-vous à vos amis - et d'autres

personnes fascinantes. Recevez des mises à jour

instantanées sur les choses qui vous intéressent. Et

regardez les événements se dérouler, en temps réel,

sous tous les angles.

Images 7.-8. <www.twitter.com> (France, pages consultées le 16/12/14).

${ }^{11}$ À ce sujet voir Musso (éd.) 2011.

11 | www.ocula.it | Giugno 2015 


\section{Ocula ${ }^{\text {flux_saggi }}$}

Les valeurs (in)différentes entre identité et image: le cas Trenitalia | Michela Deni

Tweets hashtag \#trenitalia $:^{12}$

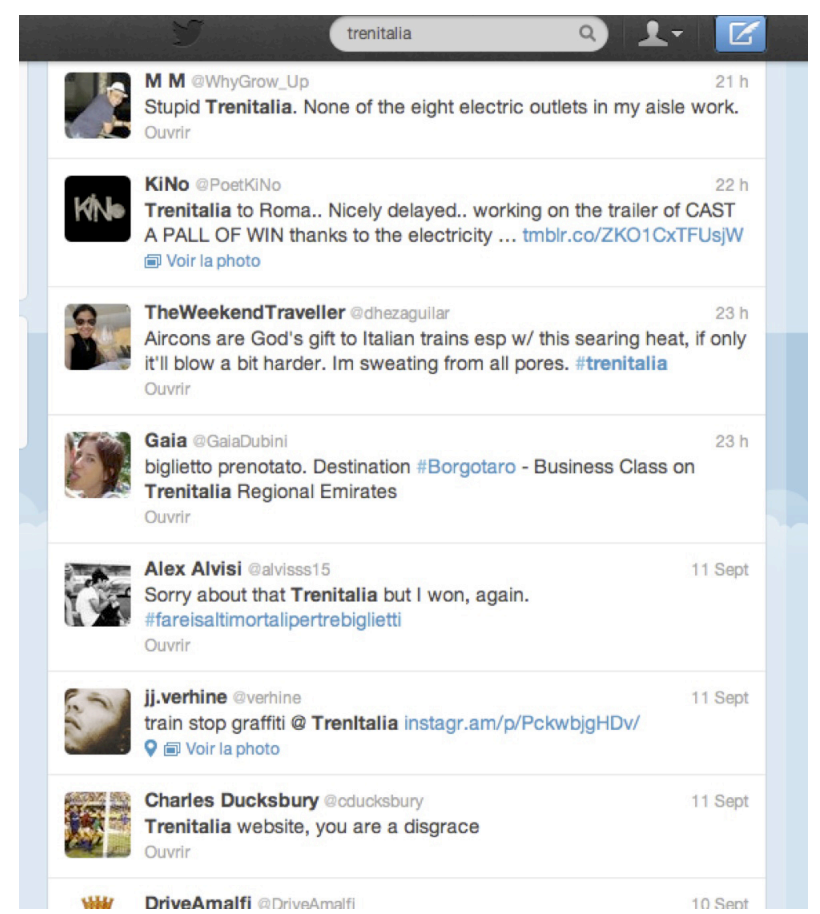

แแ⿰纟勺 DriveAmalfi $@$ DriveAmalfi

Terry Nesti eVenditoredifumo

$24 \mathrm{~min}$

Si parte con 5 minuti di ritardo \#trenitalia \#Udine \#Venezia roba che il ministro dei trasporti giapponese, Maritarda Trenytalya, si suicida Ouvrir

8

Lorenzo Zen @LorenzoZer

Peggio di qua.solo \#Trenitalia. 45 minuti di ritardo. E mancano

quelle 20-30 persone da passare. \#SaltoDelPranzo \#MiFaSoloBene Ouviris

Carlos $₫$ CarlosG_Iscar

Empezamos con los retrasos en \#Trenltalia... De momento $10 \mathrm{~min}$,

esperemos que no sea más.

Ouvir

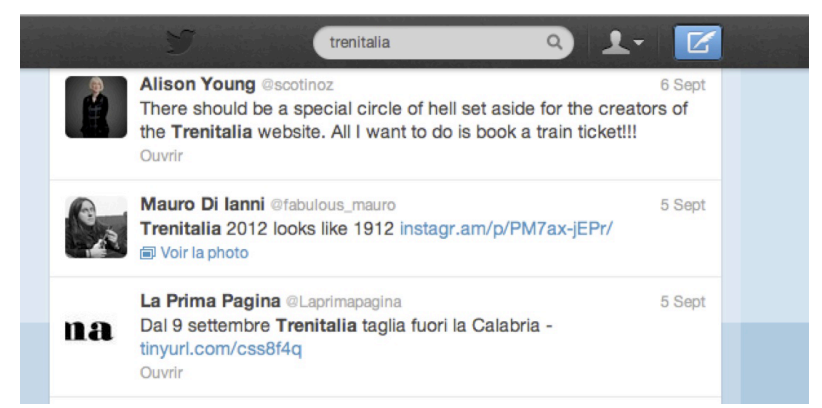

12 Twitter, pages consultées aux dates: 5, 6, 11/9/2012.

12 | www.ocula.it | Giugno 2015 


\section{Ocula $a_{\text {flux_saggi }}$}

Les valeurs (in)différentes entre identité et image: le cas Trenitalia | Michela Deni

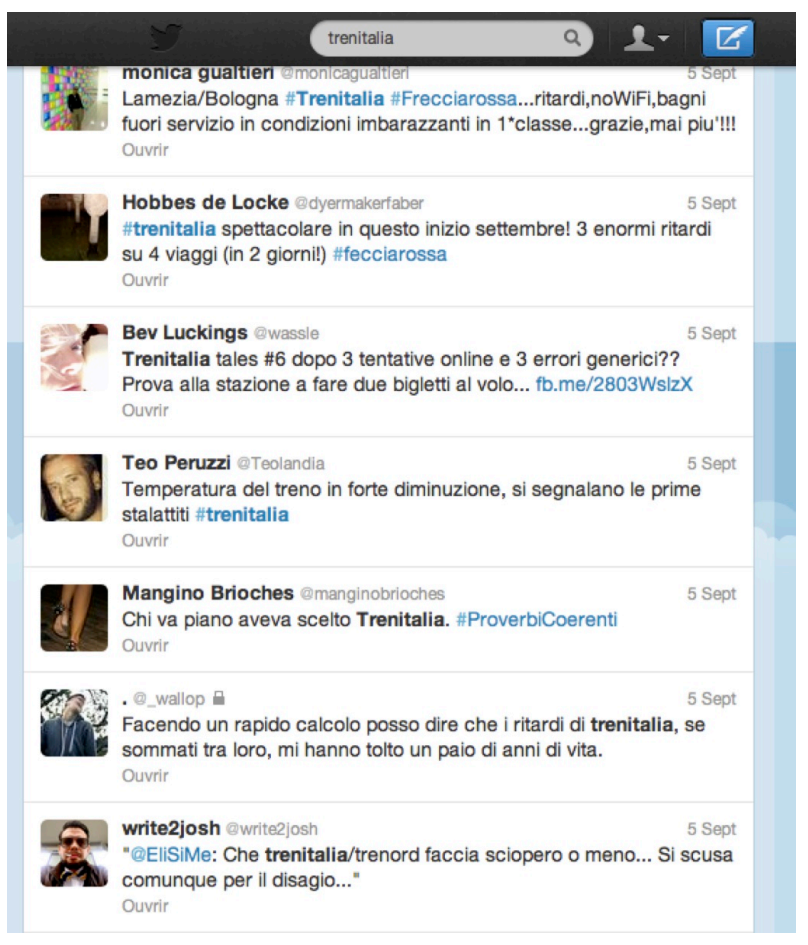

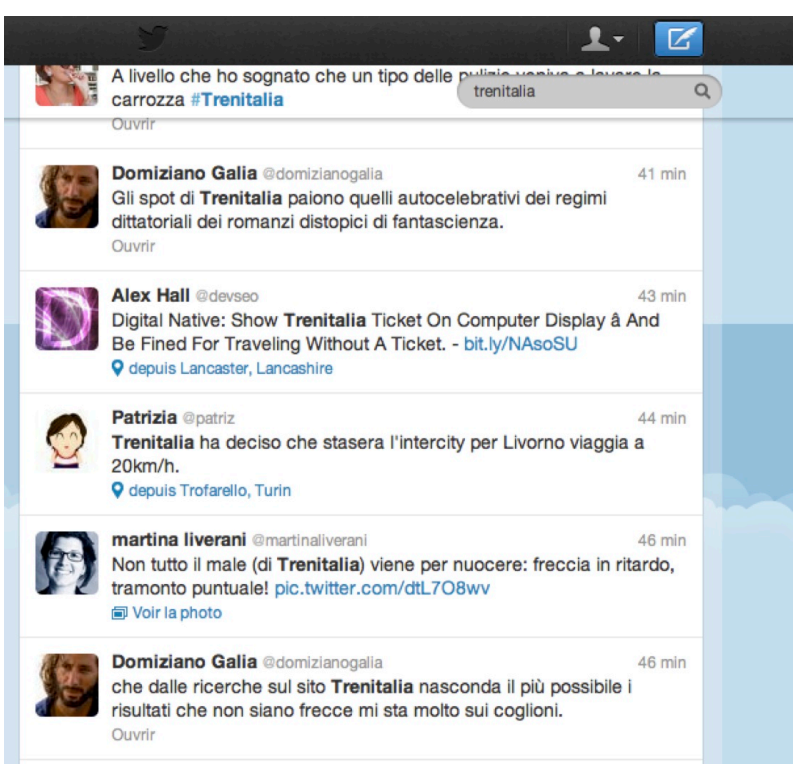

Images 9.-13. \#trenitalia <www.twitter.com> (France, pages consultées aux dates: 5, 6, 11/9/ 2012). 


\section{Oculartax_saggi}

Les valeurs (in)différentes entre identité et image: le cas Trenitalia | Michela Deni

\section{Conclusions}

C'est donc à partir de ces quelques exemples que nous pouvons comprendre clairement à quel point les valeurs sur lesquelles se fonde l'identité d'une entreprise sont prises au sérieux par les citoyens car, ces valeurs, une fois énoncées et répétées par les moyens de communications, les voyageurs s'attendent légitimement à des produits et des services qui devraient suivre. Cela montre que, dans le cas de Trenitalia et de toute entreprise dont l'écart entre l'identité et l'image est important, les sujets concernés par le contrat de confiance proposé perçoivent nettement le déséquilibre impliquant l'engagement réciproque. En fait, un contrat est l'établissement $d$ '" une relation intersubjective qui a pour effet de modifier le statut des sujets » (entrée 'Contrat' Greimas, Courtés 1979), ${ }^{13}$ le cas échéant un engagement réciproque où une entreprise promet un service à un client payant. La proposition d'un service de voyage implique alors un échange bilatéral dans lequel à l'achat du titre de transport correspond un service conséquent par rapport aux promesses communiquées par l'entreprise.

Même si elles sont établies par des lois, ces règles implicites du commerce constituent les bases sur lesquelles les voyageurs de Trenitalia ancrent leur déception légitime face à l'entreprise. Or cette entreprise incarne encore aujourd'hui, à travers ses manifestations, un modèle désuet datant du dernier siècle, typique du régime de monopole et dans lequel les transports étaient un privilège payé par le client mais accordé grâce aux investissements de l'état.

Revenant au statut de sujets concernés par le contrat, on peut observer, dans l'exemple de Trenitalia, qu'il existe un problème de connaissance et de reconnaissance de ces sujets ainsi que des valeurs qui les animent: d'une part l'entreprise ne s'engage pas dans la compréhension de ses clients et du contrat qu'elle vise à établir, d'autre part les voyageurs contemporains sont conscients de toutes les implications dérivant de leur rôle de bénéficiaires d'un service.

En d'autres termes, les valeurs que les clients de Trenitalia constatent sont donc différentes par rapport à celles déclarées par l'entreprise tandis que pour la direction de l'entreprise ces valeurs d'image deviennent indifférentes en rendant le contrat de confiance dépourvu de valeur.

13 « Le fait est que l'établissement de la structure intersubjective est en même temps, d'un côté, une ouverture sur l'avenir et sur les possibilités de l'action, et, de l'autre, une contrainte qui limite d'une certaine manière la liberté de chacun de sujets. ", entrée « Contrat » Greimas, Courtés 1979) 


\section{Ocula $a^{\text {Flux__aggi }}$}

Les valeurs (in)différentes entre identité et image: le cas Trenitalia | Michela Deni

\section{Trenitalia?}

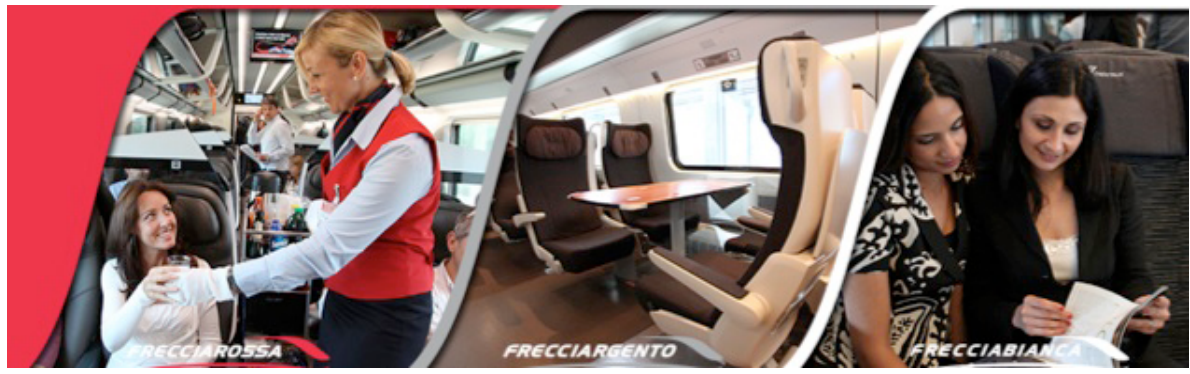

Image 14. http://www.trenitalia.com/ (page consultée le 16/12/14).

\section{Trenitalia.}

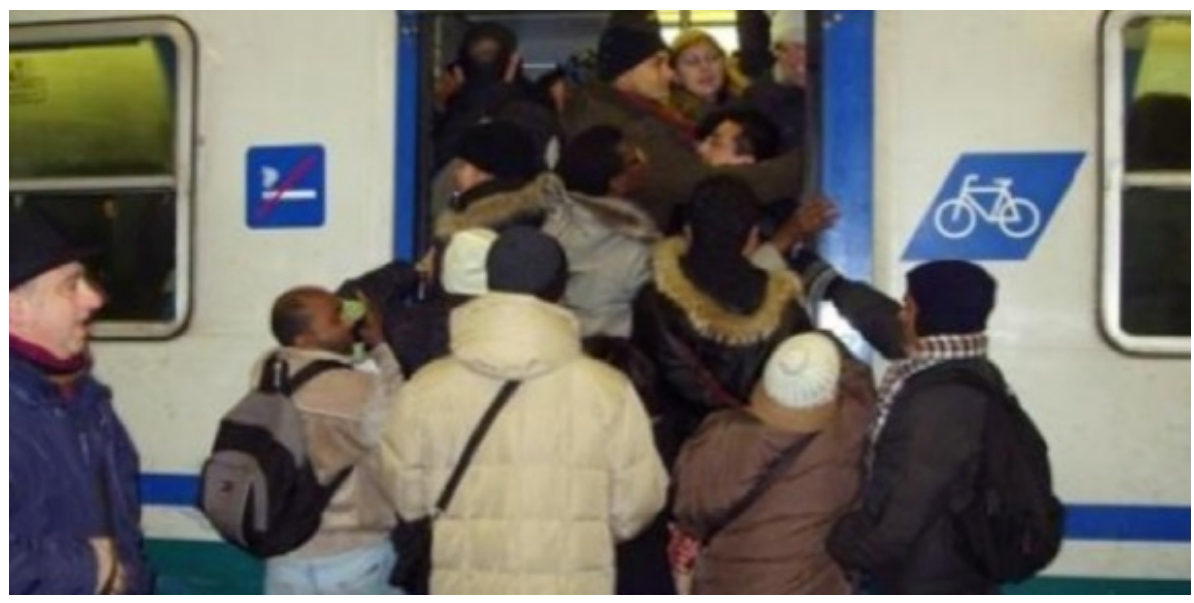

Image 15.

<valdarnopost.it/valdarno-dossier/treni-e-pendolari-quando-il-disagio-e-all-ordine-del-giorno>.

Les valeurs (in)différentes.
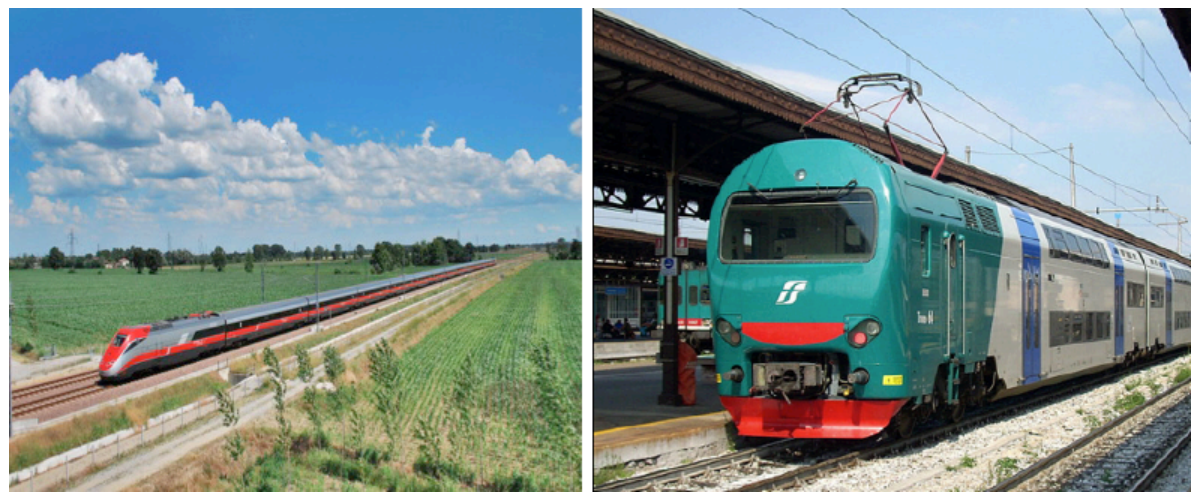

Images 16-17. <it.wikipedia.org/wiki/Treno_Alta_Frequentazione> 


\section{Ocula ${ }^{\text {flux_saggi }}$}

Les valeurs (in)différentes entre identité et image: le cas Trenitalia | Michela Deni

\section{Bibliografie}

BALMER, John M. T., GrEYSER, Stephen A.

2002 Managing the multiple identities of the corporation, University of Bradford: School of Management.

2003 Revealing the corporation: perspectives on identity, image, reputation, corporate branding, and corporate-level marketing: an anthology, London; New York: Routledge.

BIANCHI, Cinzia, RAGONESE, Ruggero

2013 L'annuncio pubblicitario, Roma: Carocci.

FABRIS, Giampaolo, MineSTRONI, Laura

2004 Valore e valori della marca. Come costruire e gestire una marca di successo, Milano: FrancoAngeli.

FLOCH, Jean-Marie

1990 Sémiotique, marketing et communication, Paris: Puf.

1995 Identités visuelles, Paris: Puf.

GREIMAS, Algirdas-J.

1970 Du sens, Paris: Seuil.

1983 Du sens II. Essais sémiotiques, Paris: Seuil.

GreIMAS, Algirdas-J., COURTÉs, Joseph

1979 Sémiotique. Dictionnaire raisonné de la théorie du langage, Paris: Hachette.

GREIMAS, Algirdas-J., COURTÉs, Joseph (éds.)

1986 Sémiotique. Dictionnaire raisonné de la théorie du langage II, Paris:

Hachette.

HEILbRunN, Benoît

2010 La marque, Paris: Puf

FABRIS, Giampaolo, MINESTRONI, Laura

2004 Valore e valori della marca. Come costruire e gestire una marca di successo, Milano: FrancoAngeli.

MARRONE, Gianfranco

2007 Il discorso di marca, Roma-Bari: Laterza.

Musso, Patrizia

2013 Slow Brand. La gestione socio-economica della marca contemporanea, Milano: FrancoAngeli.

Musso, Patrizia (ed.)

2011 Brand Reloading. Nuove strategie per comunicare, rappresentare e raccontare la marca, Milano: FrancoAngeli.

SEMPRINI, Andrea

2005 La marque, une puissance fragile, Paris: Vuibert (trad. it. La marca postmoderna. Potere e fragilità della marca nelle società contemporanee, Milano: FrancoAngeli, 2006). 